\title{
Detection and Identification of Mass Structure in Digital Mammogram
}

\author{
Prakash Bethapudi \\ Member IEEE \\ Assistnt Professorst \\ Dept of CSE \\ GITAM University
}

\author{
E.Sreenivasa Reddy \\ Professor \\ Dept of CSE \\ Nagarjuna University
}

\author{
Y.Srinivas \\ Professor \\ Dept of IT \\ GITAM University
}

\begin{abstract}
Breast Cancer is the rampant issue facing by most of the women these days. Mammography is the most successful modus operandi for verdict of breast cancer. Radiologists view mammograms to perceive the abnormalities. In this paper, we urbanized an algorithm to isolate and extract the malignant masses in mammograms for detection of breast cancer. This exertion is based on the following course of action: (a) Confiscate the background information from the DICOM image. (b)Refurbish the RGB image to gray image (c) Apply thresholding to remove the background information (ROI). (d)Apply median filter on the image to reduce random noise and preserve the edges. (e)Extract the binary image contours. (f)Carry out Area-open on the image and. (g)To end with act upon filling on the resultant image. This method was tested over the real time images of various patients taken from a cancer hospital and implemented using Matlab code. Thus, capable in executing the pre-processed image efficiently and detected the segmentation region which helped in retrieving the mass present in the digital mammogram and hence documented the malignant mass by calculating different features like Area, perimeter, Compactness, Width, Height, Isotropic factor and Eccentricity of the extracted mass.
\end{abstract}

\section{Keywords}

Contour, Compactness, Eccentricity, Height, Isotropic, Malignancy, Mammogram, Mass, Perimeter, ROI, Segmentation, Thresholding, Width.

\section{INTRODUCTION}

The American Cancer Society (ACS) reported that the majority of the women living in United States have been diagnosed and treated for breast cancer [1]. The testimony says that one in eight women will develop breast cancer during their life time. Approximately 13,000 women die from breast cancer in England each year [2]. A huge number of mammograms are taken by the breast screening program and these mammograms are examined by the experts to detect abnormalities. The UK breast screening program alone generates 1.5 million mammograms per year[3].Detection and treatment of breast cancer are most significant methods of reducing Mortality and significantly increasing the chances of survival before the cancer spreads to the other organs. This breast screening program reduced mortality caused by breast cancer in women aged 50-69 years by nearly $21.3 \%$ [4] - [6]. A CAD system for detecting malignant masses usually consists of multiple stages [7] - [8]. In the first stage, a set of candidate mass locations were detected based on image feature extracted locally. Previous research incorporated information based on regions segmented in a region of interest in a contra lateral view [9]. Because there is not always a relevant region present that can be adequately segmented, contextual features cannot always be computed in this way. In the recent work [10], the mass region was identified using morphological operations, but with a loss of some data and the edges. In the proposed algorithm, we identified the mass with perfect edges and various features of the mass have been calculated for better identification of mass and edges without any loss of data. DICOM screened images of various patients are considered for detection of masses. Details of the patient are present on the image are more important for the radiologists in performing their diagnosis. To proceed further with the work first we should eliminate the patient details present on the DICOM image. Masses in mammograms and their features greatly vary in their size and shapes. The type of cancer is identified based on the values obtained by different features. The present study is focused on segmentation of breast and detecting the masses. This paper is organized as follows. Section - II highlights the methodology to identify the masses in the breast. Section - III highlights the methodology for identification of the shape of the mass. Section - IV deals with the results and conclusion of the work.

\section{METHODOLOGY TO IDENTIFY THE MASS IN THE BREAST}

In this section we proposed a methodology which helps in the identification of masses in the breast that helps to detect the shape of the mass which helps the doctors to have a finite details and plan for effective treatment. In this methodology thee identified breast cancer patients data collected from the cancer hospital is taken into consideration. The images under this study are DICOM images. The methodology proposed is presented in the Algorithm below.

\subsection{Algorithm:}

2.1.1 Convert the given DICOM image into 'JPG' format.

2.1.2 Convert the 'JPG' image to gray scale image.

2.1.3 In order to remove the details of the patients textual

data incorporated at the time of scanning, background separation is carried out where multiple filtering techniques are used.

2.1.4 Apply thresholding so as to convert the gray image into binary image.

2.1.5 Identify the masses in the breast

2.1.6 Apply the morphological algorithms to have the final details. 


\subsection{Methodology}

In this methodology we have considered a thresholding technique based on the approach of the identification of ' $\mathrm{T}$ ' values, where the ' $T$ ' value is obtained using the matrix value generated by using an 'imtool' and selecting that value where the maximum repetation of the matrix value pertaining to the text is available. This value so selected is applied on the text data such that the value greater than the threshold value is considered as 1 and the value below the threshold value is considered as 0 . Hence using this process a matrix is generated which consists of two classes, a class containing black pixels and the class containing the white pixels. This class of white pixels will be generally the tissue which in this is a breast. In order to enhance the image quality repetitive application of median filter is carried out so that the blur inside the image is minimized and the image is processed. The outputs obtained by the above methodologies are depicted in the following figures
Fig.1DICOM Image

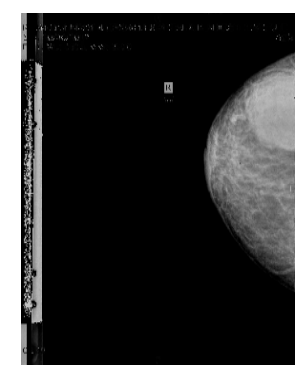

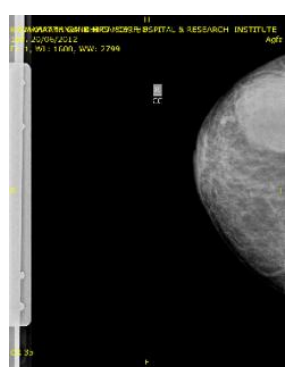

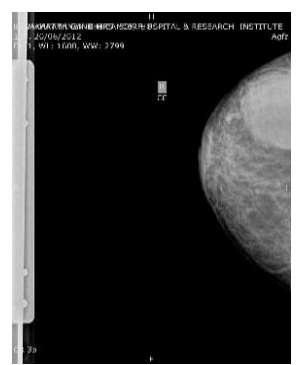

Fig.2 Gray-scale Image

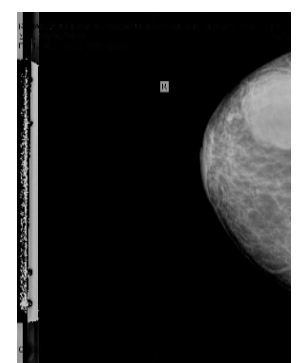

Fig.3 After Thresholding Fig.4Preprocessed Image

The algorithm for the identification of threshold and median filter are presented in the below

Apply Thresholding on gray scale image. Fig.3

$$
\begin{aligned}
& \text { for } i=1: m \\
& \text { for } j=1: n \\
& \text { if } b(i, j)>198 \\
& c(i, j)=0 \\
& \text { else } \\
& c(i, j)=b(i, j) \\
& \text { end }
\end{aligned}
$$

end

end

Preprocessing using Median Filter. Fig.4

$$
\begin{aligned}
& \text { for } \mathrm{i}=2: \mathrm{m}-1 \\
& \text { for } \mathrm{j}=2: \mathrm{n}-1 \\
& \mathrm{~g}=[\mathrm{c}(\mathrm{i}-1, \mathrm{j}-1) \quad \mathrm{c}(\mathrm{i}-1, \mathrm{j}) \mathrm{c}(\mathrm{i}-1, \mathrm{j}+1) ; \ldots \\
& c(i, j-1) \quad c(i, j) c(i, j+1) ; \ldots \\
& \mathrm{c}(\mathrm{i}+1, \mathrm{j}-1) \mathrm{c}(\mathrm{i}+1, \mathrm{j}) \mathrm{c}(\mathrm{i}+1, \mathrm{j}+1)] \text {; } \\
& \text { b1 }(\mathrm{i}-1, \mathrm{j}-1)=\operatorname{median}(\operatorname{median}(\mathrm{g})) \text {; } \\
& \text { end }
\end{aligned}
$$

The extracted 'jpg' image of the patient which is pre processed and converted into gray scale is considered and the boundaries are to be extracted for the future analysis. In order to identify the boundary pixels we have considered a seed pixel inside the image region and identified its neighborhood using 4 connections. These regions so obtained using 4 - connection are considered to be the boundary pixels. The boundaries that are extracted are to be filled so as to overcome the holes inside the image regions. Morphological operations open and close are used to fill the gaps in the holes inside the image region. The result obtained are presented in the below figures 5 to 8 and the algorithms used are given below

1. Determine connected components $\mathrm{L}=$ bwlabeln(bw,conn);

2. Compute the area of each component $\mathrm{S}=$ regionprops(L,'Area');

3. Remove small objects Bw2=ismember $($ L, find $([$ S.Area $]>=p))$;

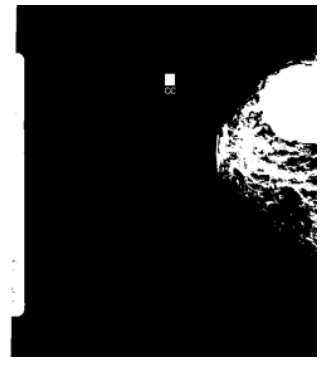

Fig.5 Perform BwAreaopen

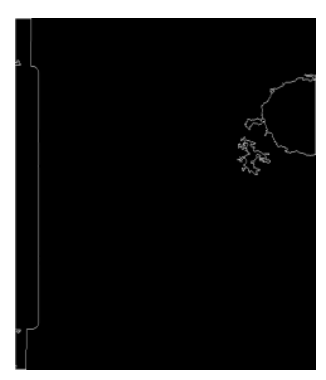

Fig.7 Remove small objects

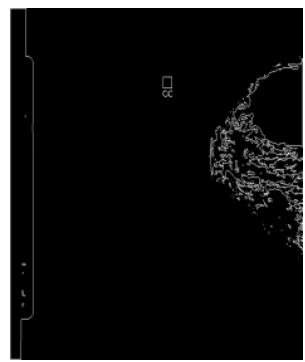

Fig.6 Perform Bwmorph

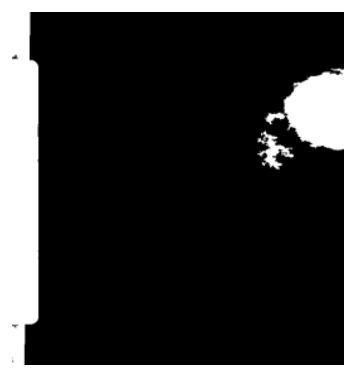

Fig.8 Mass structure-imfill

\section{METHODOLOGY TO IDENTIFIY THE SHAPE OF THE MASS}

Once the masses are identified, in order to facilitate the experts to have an overview of the shape of masses, mathematical techniques such as identifying the area is executed. It helps in understanding the area covered by a particular mass. The process of identification is given below.

1. Area : This express the extent of a $2 d$ surface or a shape.

$$
\begin{aligned}
& A=0 ; \\
& \text { for } i=1: m \\
& \text { for } j=1: n \\
& \text { If } d 1(I, j)==1 \\
& A=A+1 ;
\end{aligned}
$$




$$
\text { end }
$$

end

Once the mass is identified the area covered is detected. The immediate goal is to specify the minimum and maximum distances, perimeter, compactness, width and height of the mass. In order to achieve this, the mathematical formulas in use are used for this purpose and are given below.

2. Isotropic Factors which specify the minimum and the maximum distance

Mass deficit coefficient MD $=100 *((\mathrm{~A} 1-\mathrm{A}) / \mathrm{A} 1)$

Mass excess coefficients $\mathrm{ME}=100 *((\mathrm{~A}-\mathrm{A} 1) / \mathrm{A})$
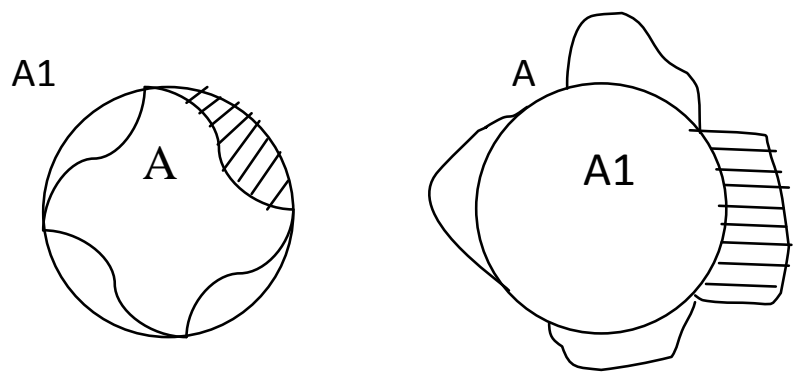

3. Perimeter is a path that surrounds a $2-\mathrm{D}$ shape., ie, it is the length of the outline of the shape.

$$
\text { Perimeter }=\operatorname{sum}(\operatorname{sum}(\mathrm{p}))
$$

4. Compactness is arranged within a relatively small space joined or packed together.

$$
(4 * \mathrm{PI} * \mathrm{~A}) /(\text { Peri })^{\wedge} 2
$$

Width calculation

$$
\begin{aligned}
& \mathrm{W}(\mathrm{i})=\operatorname{sum}(\mathrm{d} 1(\mathrm{i},:)) ; \\
& \mathrm{W} \_\max =\max (\mathrm{w}) ;
\end{aligned}
$$

Height Calculation

$$
\begin{aligned}
& \mathrm{H}(\mathrm{j})=\operatorname{sum}(\mathrm{d} 1(:, \mathrm{j})) ; \\
& \text { H_max }=\max (\mathrm{h}) ;
\end{aligned}
$$

5. Eccentricity is the measure of how much of conic section deviates from being circular. ie, the amount by which its orbit around another body deviates from a perfect circle. The exactness of the shape can only be achieved through eccentricity which helps in proper identification of the shape of the mass. Eccentricity is based on the identification of the focus region and is calculated by using the formula

$$
\text { ( W_max / H_max ); }
$$

Where W_max is the width of the mass and H_max is the height of the mass. From the eccentricity the shape of the objects are identified.: If the eccentricity $=0$ it resembles that the shape of the mass is circular. If the value is between 0 and 1 , the shape is elliptical. If the value is 1 , it is parabola. If the value is between 1 and 2 , it is hyperbola. If the value is 2 , it is a hyperbola. If the value is greater than 2 , it is diretrix. The various shapes of the curves based on eccentricity is shown in the below figure.

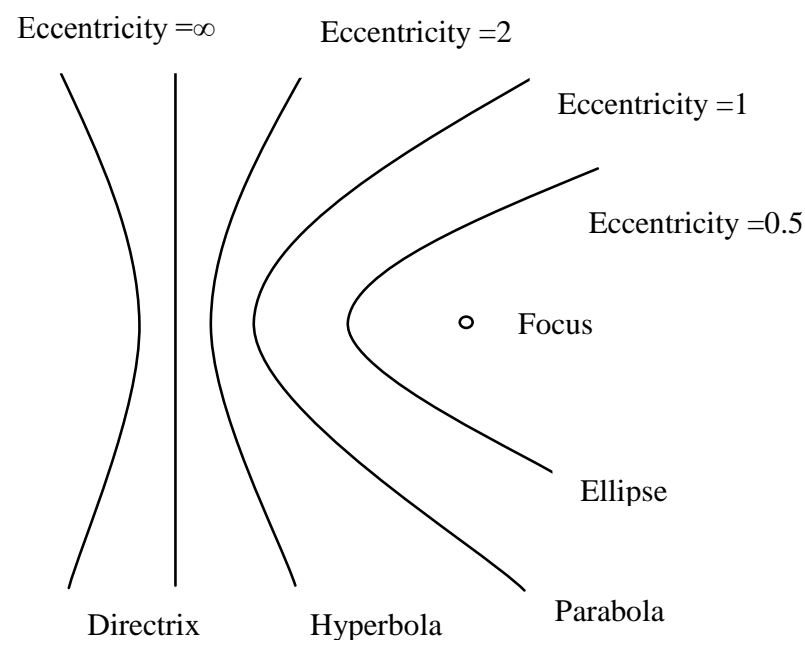

Fig.9 Eccentricity shapes and ranges

The obtained measures of the detected mass are shown below

Table.1

\begin{tabular}{|c|c|c|}
\hline S.No & Parameters & Value \\
\hline 1 & Area & 3.5207 \\
\hline 2 & Perimeter & 0.2226 \\
\hline 1 & Compactness & 0.0893 \\
\hline 4 & Eccentricity & 0.3867 \\
\hline
\end{tabular}

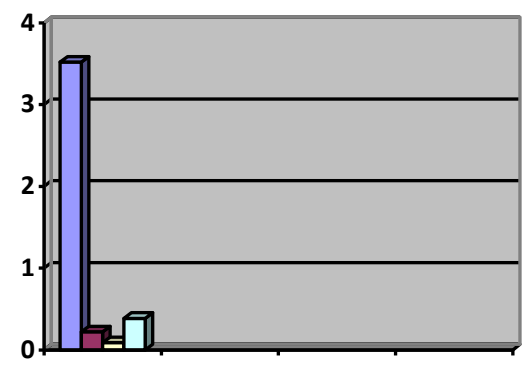

\begin{tabular}{|l|}
\hline Area \\
$\square$ Perimeter \\
$\square$ Compactness \\
$\square$ Eccentricity
\end{tabular}

The results obtained for different features have been displayed in the graph shown above.

\section{RESULT AND CONCLUSION}

In this paper, a novel methodology is presented for the identification of the structure of the mass in a breast cancer patient. Various parameters for extraction and identification of the shape of mass presented in section III and shown in Table1 concludes that, for the considered breast cancer image the area of the mass is 3.5207. From the above results it can be clearly shown that the Perimeter of the mass is 0.2226 , the 
Compactness of the mass is 0.0893 and the eccentricity of the mass for this image under consideration is 0.3867. This resembles the shape of the mass depicted in the breast which is assumed to be a malignant and is ellipse in shape shown in fig.9. This methodology felicitates the oncologist's to plan for further processing and effective treatment.

\section{ACKNOWLEDGEMENTS}

Our sincere thanks to Dr.Madhuri.P, Consultant, Radiologist, Mahatma Gandhi Cancer Hospital, Visakhapatnam, AP, INDIA., for providing us original screened breast images in carrying out our research work. Thanks to the experts who have contributed towards the development of this template.

\section{REFERENCES}

[1]. American Cancer Society(2009a). Breast cancer facts and figures 2009-2010. Atlanta: American cancer society, Inc.

[2]. N.B.C.S.P.W site.(2005).[Online].Available at : www.cancerscreening.nhs.uk/breastscreen

[3]. R.Zwiggelaar,T.CParr,J.E.Schumm,I.W.Hutt,C.J.Taylor, S.M.Asley and C.R.M.Boggis, "Model based detection of peculated lesions in mammograms"Med Image Anal.,Vol.3, no.1,pp39-62,1999.

[4]. E.L Thurfjell and J.A.Lindgren, "Breast cancer survival rates with mammographic screening : Similar favourable survivia rates for women younger and those older than 50 years” Radiology, vol.201,no.2,pp.421-426, 1996.

[5]. P.B.Dean, "Overview of breast cancer screening" in proc.Digital Mammography 1996.Amsterdam,The Netherlands: Elsevier,pp19-26

[6]. I.Andersson,"Breast cancer screening results" in Proc.Digital mammography 1998.Norwell, MA: Kulwer, pp 427-433.

[7]. J.Wei,B.Sahiner,L.Hadjiiski, H.Chan, N.Petrick, M.Helvie, M.Roubidoux, J.Ge and C.Zhou, " Computer - aided detection of breast masses on full field digital mammograms,"Med.Phys.,vol.32,no.9,pp.28272838,2005 .

[8]. B.Zheng,J.Leader,G.Abrams,A.Lu,L.Wallace,G.Maitz and D.Gur, "Multiview - based computer - aided detection scheme for breast masses,"Med.Phys., vol.33,no.9,pp.3135-3143,2006.

[9]. Y.Wu,J.Wei,L.Hadjiiski,J.Wei,C.Zhou,B.Sahiner,J.Ge,J. Shi,Y.Zhang and H.Chan, "Bileteral analysis based false positive reduction for computer - aided mass detection," Med.Phys., vol.34,no.8,pp.3334-3344.

[10].Prakash Bethapudi, Dr.E.Srinivasa Reddy, Dr.Madhuri.P, "Detection of Malignancy in digital mammograms form segmented breast region using morphological techniques,”IOSR-JEEE,vol.5,Issue.4, pp.09-12,2013. 